\title{
Disseminated Varicella-zoster Virus Infection Causing Fatal Pneumonia in an Immunocompromised Patient with Chronic Interstitial Pneumonia
}

\begin{abstract}
Hiroshi Ueno ${ }^{1}$, Masachika Hayashi ${ }^{1}$, Shun Nagumo ${ }^{1}$, Kosuke Ichikawa ${ }^{1}$, Nobumasa Aoki ${ }^{1}$, Yasuyoshi Ohshima ${ }^{1}$, Satoshi Watanabe ${ }^{1}$, Toshiyuki Koya ${ }^{1}$, Tatsuya Abé ${ }^{2,3}$, Riuko Ohashi ${ }^{3,4}$,

Yoichi Ajioka ${ }^{3}$ and Toshiaki Kikuchi ${ }^{1}$
\end{abstract}

\begin{abstract}
:
Viral pneumonia caused by varicella-zoster virus (VZV) infection is a rare but important complication, especially regarding varicella infections. Although disseminated cutaneous herpes zoster (DCHZ) is often associated with visceral diseases, there have been few reports of DCHZ-related pneumonia. We herein report a rare case of a lethal disseminated VZV infection that caused severe pneumonia in a Japanese patient who had chronic interstitial pneumonia. Physicians should consider the possibility of VZV-related pneumonia, especially in patients with a medical history of hematopoietic stem cell transplantation and immunosuppressive therapy.
\end{abstract}

Key words: viral pneumonia, varicella-zoster virus, disseminated cutaneous herpes zoster, myelodysplastic syndrome, chronic interstitial pneumonia

(Intern Med 60: 1077-1082, 2021)

(DOI: 10.2169/internalmedicine.5396-20)

\section{Introduction}

Primary infection with varicella-zoster virus (VZV), varicella, or chickenpox usually presents as a generalized vesicular rash and it can sometimes cause pneumonia (1-3). Pneumonia is the most frequent serious complication of disseminated VZV infection and it has a high mortality rate (1). Herpes zoster is another type of VZV infection which is caused by the reactivation of latent VZV with the spread of the virus along the sensory nerve to the dermatome (3). Disseminated cutaneous herpes zoster (DCHZ) is defined by the presence of 20 or more vesicular lesions outside the primary and adjacent dermatomes and is characterized by viremia of VZV as well as of varicella (4). Therefore, DCHZ can sometimes include visceral involvement, such as an abdominal infection $(4,5)$, but unlike with varicella, pneumonia is a rare complication of DCHZ. We herein report a rare case of a lethal disseminated VZV infection that caused severe pneumonia in a Japanese patient who had chronic interstitial pneumonia. This case serves to remind physicians that VZV reactivation can sometimes induce severe pneumonia, so the possibility of VZV-related pneumonia must be considered in immunocompromised patients.

\section{Case Report}

A 68-year-old Japanese man with a 3-year history of chronic interstitial pneumonia developed acute-onset leftside chest and shoulder pain associated with shortness of breath. He presented to our department a few days after the

\footnotetext{
${ }^{1}$ Department of Respiratory Medicine and Infectious Diseases, Niigata University Graduate School of Medical and Dental Sciences, Japan, ${ }^{2}$ Division of Oral Pathology, Graduate School of Medical and Dental Sciences, Niigata University, Japan, ${ }^{3}$ Division of Molecular and Diagnostic Pathology, Graduate School of Medicine and Dental Sciences, Niigata University, Japan and ${ }^{4}$ Histopathology Core Facility, Niigata University Faculty of Medicine, Japan

Received: May 21, 2020; Accepted: September 23, 2020; Advance Publication by J-STAGE: November 9, 2020

Correspondence to Dr. Masachika Hayashi, mhayashi@pk9.so-net.ne.jp
} 
Table. Baseline Laboratory Findings on Admission to Our Hospital.

\begin{tabular}{|c|c|c|c|}
\hline Hematology & \multicolumn{3}{|c|}{ Biochemistry } \\
\hline WBC & $10,260 / \mu \mathrm{L}$ & AST & $40 \mathrm{IU} / \mathrm{L}$ \\
\hline Neut & $82.5 \%$ & ALT & $32 \mathrm{IU} / \mathrm{L}$ \\
\hline Lymp & $8.2 \%$ & LDH & $426 \mathrm{IU} / \mathrm{L}$ \\
\hline Mono & $8.0 \%$ & ALP & $256 \mathrm{IU} / \mathrm{L}$ \\
\hline Eosi & $1.1 \%$ & rGTP & $26 \mathrm{IU} / \mathrm{L}$ \\
\hline Baso & $0.2 \%$ & $\mathrm{CK}$ & $35 \mathrm{IU} / \mathrm{L}$ \\
\hline $\mathrm{RBC}$ & $547 \times 10^{4} / \mu \mathrm{L}$ & $\mathrm{TP}$ & $7.2 \mathrm{~g} / \mathrm{dL}$ \\
\hline $\mathrm{Hb}$ & $18.6 \mathrm{~g} / \mathrm{dL}$ & ALB & $4.1 \mathrm{~g} / \mathrm{dL}$ \\
\hline $\mathrm{HCT}$ & $53.9 \%$ & T-Bil & $1.0 \mathrm{mg} / \mathrm{dL}$ \\
\hline \multirow[t]{2}{*}{ PLT } & $12 \times 10^{4} / \mu \mathrm{L}$ & BUN & $41 \mathrm{mg} / \mathrm{dL}$ \\
\hline & & Cre & $1.33 \mathrm{mg} / \mathrm{dL}$ \\
\hline Serology & & $\mathrm{Na}$ & $135 \mathrm{mEq} / \mathrm{L}$ \\
\hline CRP & $3.86 \mathrm{mg} / \mathrm{dL}$ & $\mathrm{K}$ & $5.0 \mathrm{mEq} / \mathrm{L}$ \\
\hline PCT & $0.24 \mathrm{ng} / \mathrm{mL}$ & $\mathrm{Cl}$ & $96 \mathrm{mEq} / \mathrm{L}$ \\
\hline ANA & $<40$ index & BG & $269 \mathrm{mg} / \mathrm{dL}$ \\
\hline Anti-dsDNA & $4 \mathrm{IU} / \mathrm{mL}$ & IgG & $714 \mathrm{mg} / \mathrm{dL}$ \\
\hline Anti-RNP & $<5.0$ index & & \\
\hline Anti-SS-A & $<5.0$ index & \multicolumn{2}{|c|}{ Arterial blood gas (ambient air) } \\
\hline Anti-SS-B & $<5.0$ index & $\mathrm{pH}$ & 7.396 \\
\hline Anti-ARS & $<5.0$ index & $\mathrm{pCO}_{2}$ & 32.4 Torr \\
\hline MPO-ANCA & $<1.0 \mathrm{U} / \mathrm{mL}$ & $\mathrm{pO}_{2}$ & 49.1 Torr \\
\hline PR3-ANCA & $<0.5 \mathrm{U} / \mathrm{mL}$ & $\mathrm{HCO}_{3}^{-}$ & $19.4 \mathrm{mmol} / \mathrm{L}$ \\
\hline$\beta$-D-glucan & $<6.0 \mathrm{pg} / \mathrm{mL}$ & $\mathrm{BE}$ & $-4.1 \mathrm{mmol} / \mathrm{L}$ \\
\hline CMV Ag & $0 / 0$ & & \\
\hline KL-6 & $4,507 \mathrm{U} / \mathrm{mL}$ & Urinalysis & \\
\hline \multirow[t]{2}{*}{ SP-D } & $632.4 \mathrm{ng} / \mathrm{mL}$ & Protein & $2+$ \\
\hline & & Sugar & - \\
\hline Mycoplasma Ab & $(-)$ & Blood & - \\
\hline Chlamydia pneumoniae IgG & $(-)$ & & \\
\hline Chlamydia pneumoniae IgA & $(-)$ & & \\
\hline Chlamydia pneumoniae IgM & $(-)$ & & \\
\hline
\end{tabular}

WBC: white blood cell, Neut: neutrophil, Lymp: lymphocyte, Mono: monocyte, Eosi: eosinophil, Baso: basophil, RBC: red blood cell, Hb: hemoglobin, HLT: hematocrit, PLT: platelet, AST: aspartate aminotransferase, ALT: alanine aminotransferase, LDH: lactate dehydrogenase, ALP: alkaline phosphatase, $\gamma$-GTP: gamma-glutamyl transferase, CK: creatine kinase, TP: total protein, ALB: albumin, T-Bil: total bilirubin, BUN: blood urea nitrogen, Cre: creatinine, Na: sodium, K: potassium, $\mathrm{Cl}$ : chlorine, BG: blood glucose, IgG: immunoglobulin G, CRP: C-reactive protein, PCT: procalcitonin, ANA: antinuclear antibody, MPO-ANCA: anti-myeloperoxidase anti-neutrophil cytoplasmic antibodies, PR3-ANCA: anti-proteinase 3 anti-neutrophil cytoplasmic antibodies, CMV Ag: cytomegalovirus antigenemia, KL-6: Krebs von den Lungen-6, SP-D: surfactant protein-D, Ab: antibody

onset of symptoms. His medical history included chronic interstitial pneumonia, hypertension, and myelodysplastic syndrome. His myelodysplastic syndrome had been maintained in complete remission without any immunosuppressant drugs after he received an HLA-matched hematopoietic stem cell transplantation from his sister 6 years previously. His chronic interstitial pneumonia had been followed for 3 years. He underwent video-assisted thoracoscopic surgery three years before admission and although we were unable to classify his chronic interstitial pneumonia then, even after expert multidisciplinary discussions, the most likely possibilities were either chronic hypersensitivity pneumonitis or idiopathic interstitial pneumonia. During the first 2 years, he did not take any medication, but he experienced an acute ex- acerbation 1 year previously. The acute exacerbation was treated by glucocorticoid pulse therapy, and we tapered the dosage in the outpatient department. He was diagnosed with diabetes mellitus and osteoporosis 1 year previously but those were well controlled. He was a previous smoker with a 20-pack year history and drank alcohol occasionally. He had no recent history of travel or frequenting hot springs, public baths, or sick contacts. He also did not have any pets. He had previously worked as a plumber but was now retired. There was no family history of interstitial pneumonia or structural pulmonary abnormalities. His regular medications were fexofenadine, montelukast, amlodipine, lansoprazole, prednisolone $(10 \mathrm{mg})$, teneligliptin, alendronic acid, and eldecalcitol. 


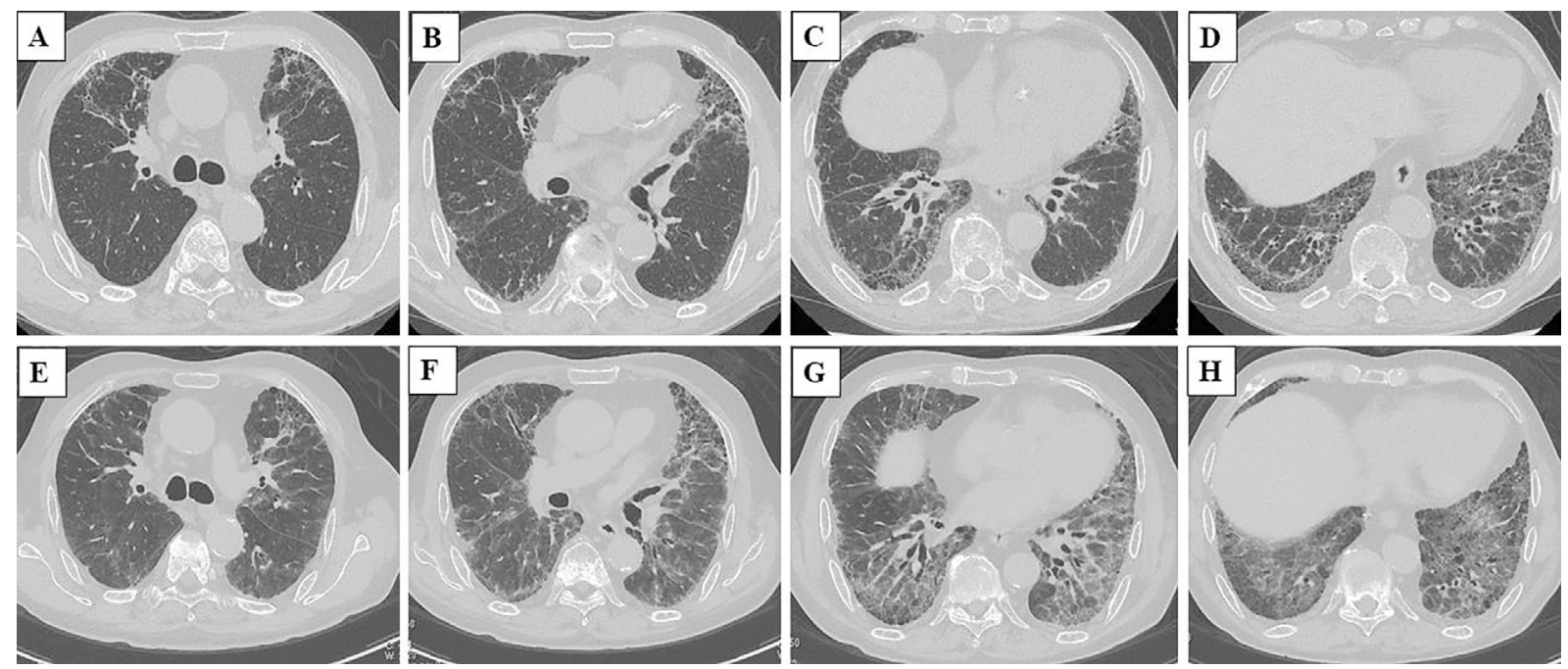

Figure 1. Chest computed tomography scan, taken 4 months before admission (A-D) and at the time of admission (E-H).

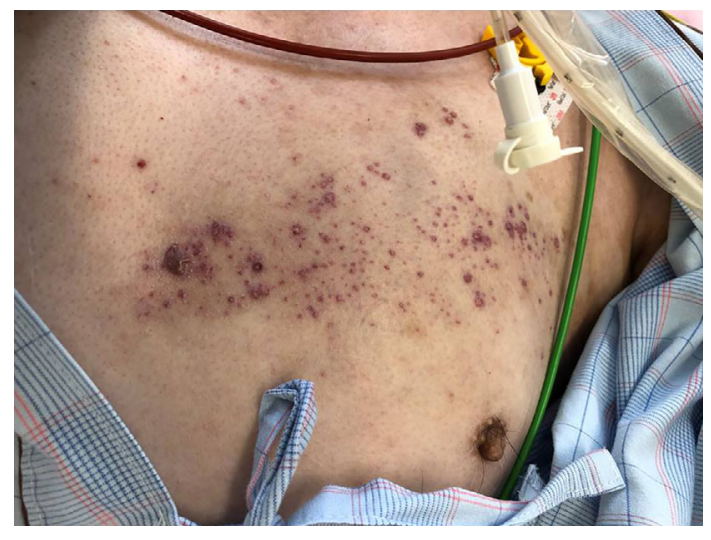

Figure 2. A diffuse vesicular rash of the chest is noted on day 3 of hospitalization.

On physical examination, he appeared ill, his body temperature was $36.0^{\circ} \mathrm{C}$, and his blood pressure was $117 / 82$ $\mathrm{mmHg}$, pulse was 101 beats/min, and respiratory rate was 24 breaths/min with an $\mathrm{O}_{2}$ saturation of $90 \%$ on $2 \mathrm{~L} / \mathrm{min}$ oxygen administration through a nasal cannula. A chest examination revealed bilateral fine crackles. There was no evidence of oral thrush. His neck was supple; the results of his cardiovascular examination were normal, and abdominal examination findings were unremarkable with no hepatosplenomegaly. The results of his neurological examination were completely unremarkable, and we did not find any skin lesions at admission.

Laboratory examination results revealed leukocytosis, erythrocytosis, renal dysfunction, type I respiratory failure, proteinuria, and hypogammaglobulinemia. His Krebs von den Lungen-6 (KL-6) and surfactant protein-D (SP-D) levels were remarkably elevated compared with his baseline values (KL-6, 2,627.6 U/mL; SP-D, $337.5 \mathrm{ng} / \mathrm{mL}$ ) (Table).

A chest X-ray showed bilateral diffuse interstitial infiltrates with a predominantly reticular pattern. A subsequent high-resolution computed tomography scan of the thorax re- vealed bilateral ground-glass opacities with interlobular and intralobular septal thickening affecting all lobes but with a middle and lower level predominance. Traction bronchiectasis had worsened compared with that 4 months before admission (Fig. 1).

Initially, the patient was diagnosed with an acute exacerbation of chronic interstitial pneumonia. Intravenous glucocorticoid pulse therapy, continuous heparin infusion, and antibiotics were started. On day 2 of his hospitalization, polymyxin B immobilized fiber column direct hemoperfusion was performed because his respiratory failure had progressed. Despite those treatments, his clinical symptoms and respiratory failure rapidly worsened, and he was intubated 3 days after admission. A large amount of hemoptysis was found in the airway during intubation so heparin administration was stopped. Multiple blisters had developed on his skin and he was diagnosed with DCHZ (Fig. 2). His sputum had a positive polymerase chain reaction (PCR) test result for $V Z V$, consistent with viral pneumonia associated with VZV. The administration of intravenous acyclovir was added immediately but his multiple organ failure worsened rapidly along with a severe hemorrhagic tendency. Despite administering intensive care, the patient died of respiratory failure 7 days after the onset of his symptoms (Fig. 3).

At autopsy, skin lesions showed positive immunostaining to anti-VZV antibody in the epidermis and intranuclear inclusion bodies were identified. The lung tissue showed the exudative to the organizing phase of diffuse alveolar damage with massive alveolar hemorrhage accompanied by swelling, multinucleation, and detachment of the alveolar epithelium. The lung tissue also showed positive immunostaining for anti-VZV antibody, consistent with the diagnosis of pneumonia due to VZV infection (Fig. 4). VZV antigen-positive cells by immunohistochemistry were further observed in the esophagus, liver, kidney, heart, spleen, urinary bladder and bone marrow. A serum sample collected at the time of blister formation was negative for VZV-IgM and positive for VZV-IgG. 


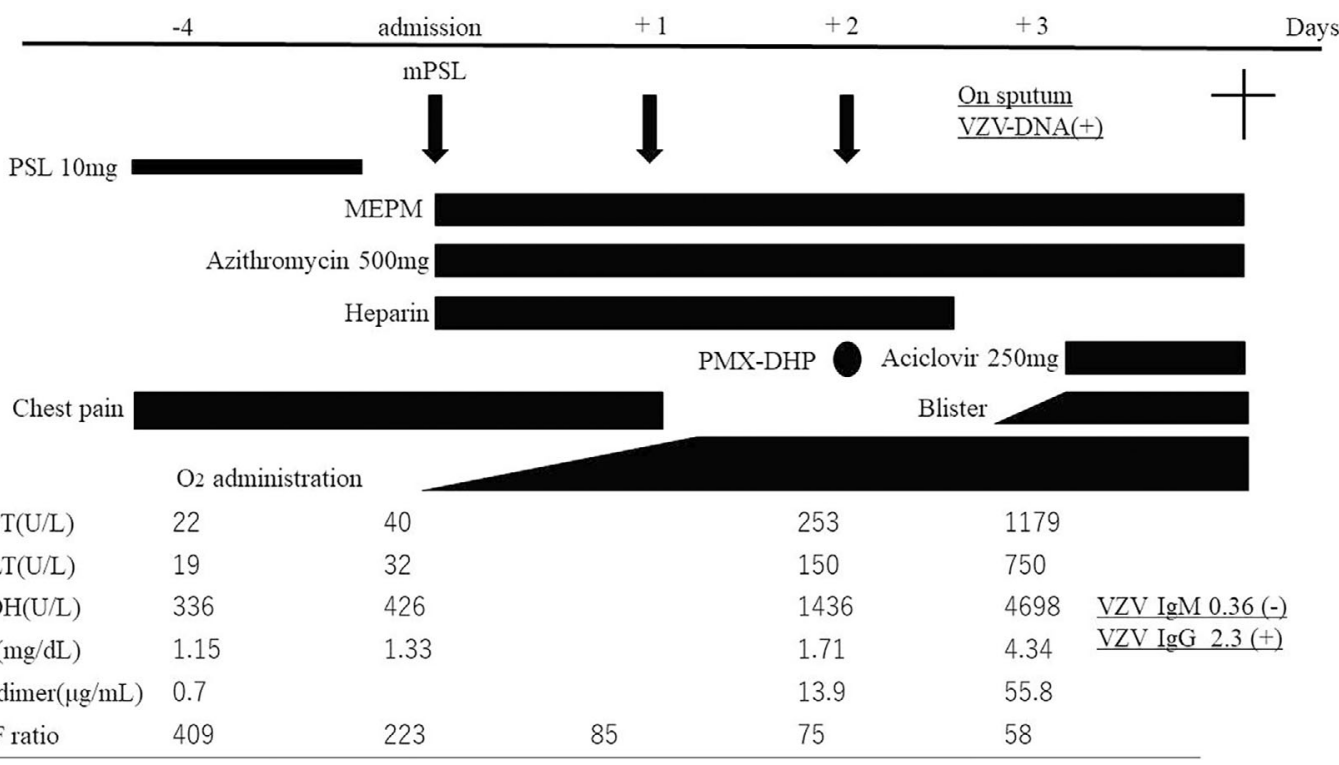

Figure 3. The patient's clinical course after presenting with chest pain.
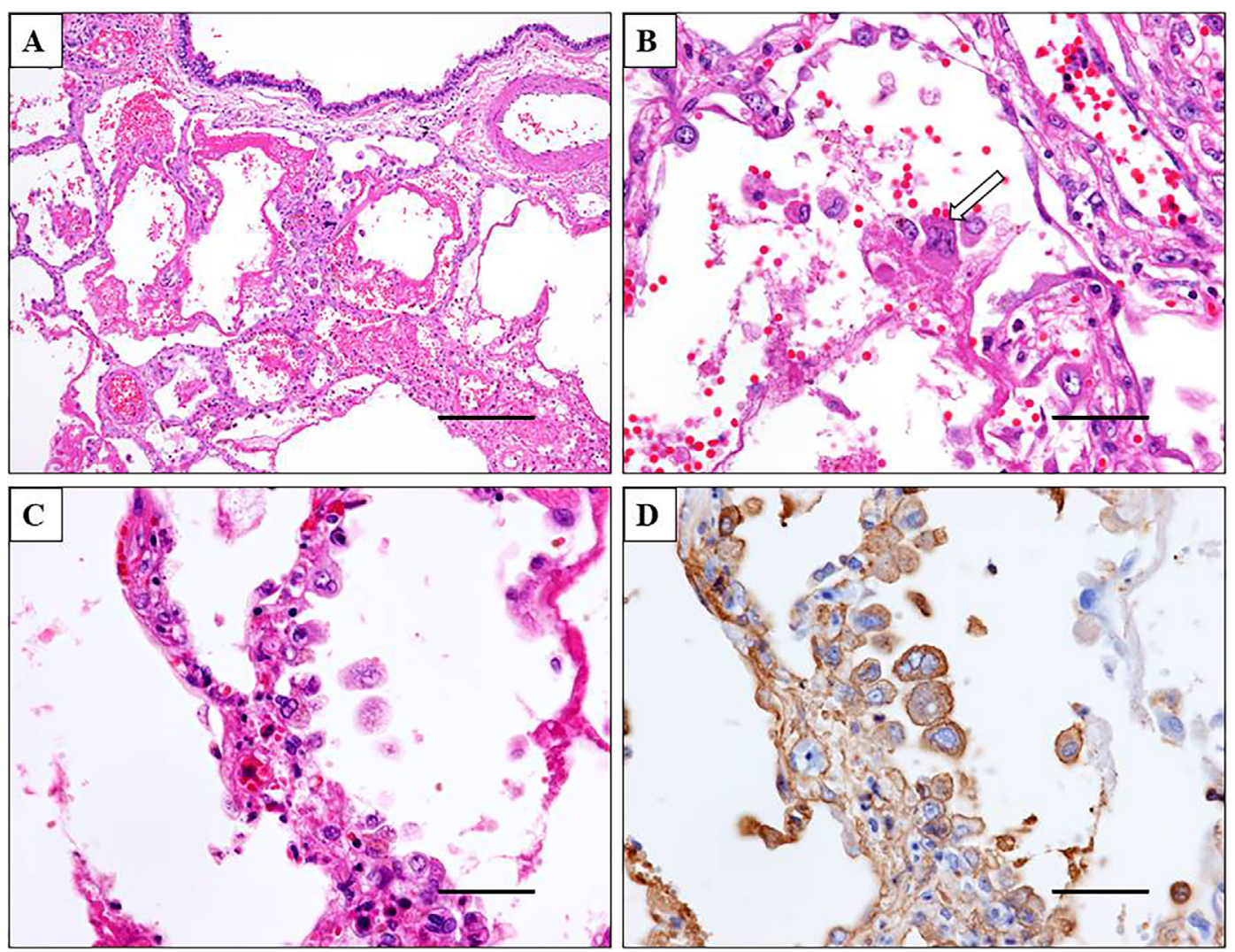

Figure 4. Histological findings of the lung at autopsy. A: Hematoxylin and Eosin (H\&E) staining shows detachment, swelling, and multinucleation of the alveolar epithelium and diffuse alveolar damage (e.g., alveolar wall edema, hyaline membranes, and alveolar hemorrhage). There are some alveolar macrophages. Scale bar, $100 \mu \mathrm{m}$. B: H\&E staining shows multinucleated giant cells with an intranuclear inclusion body (arrow). Scale bar, $50 \mu \mathrm{m}$. C, D: H\&E staining (C) and immunostaining with anti-varicella-zoster virus antibodies (D). Positive staining for varicella-zoster virus antigens observed in the alveolar epithelium. Scale bars, $50 \mu \mathrm{m}$ 


\section{Discussion}

We herein reported a case of severe pneumonia caused by VZV infection in a Japanese patient with chronic interstitial pneumonia who was receiving oral prednisolone at a dose of $10 \mathrm{mg}$ daily.

$\mathrm{VZV}$ is one of eight herpes viruses known to cause human infection and it is distributed globally. There are two types of VZV infection: primary VZV infection (often called varicella or chickenpox) and herpes zoster, which is caused by a reactivation of the virus $(1,3)$. VZV infection is generally considered self-limiting but fatal infections can sometimes occur, especially in immunocompromised patients. Pneumonia is the most common and serious complication of VZV infection, and it is usually caused by varicella and not herpes zoster. A recent retrospective cohort study of 102 patients with VZV-induced pneumonia from 1,996 to 2,015 in 29 French intensive care units reported an overall mortality rate of $24 \%$, which increased to $43 \%$ in patients who were administered invasive mechanical ventilation (6). The authors considered that the patients had primarily experienced an unfortunate complication of varicella rather than herpes zoster. Only a few case reports have described pneumonia caused by herpes zoster infection (7), and therefore herpes zoster-related pneumonia appears to be extremely rare and it is therefore not well known. DCHZ is an advanced type of herpes zoster that represents viremia of VZV as well as varicella, and our patient was diagnosed with DCHZ by a dermatologist. The common radiographic findings of VZV pneumonia are ill-defined nodular or reticular densities of various sizes scattered throughout both lungs $(1,2)$. Our patient showed bilateral ground-glass opacities with interlobular and intralobular septal thickening affecting all lobes and there were no nodular changes, so his radiographic findings were completely different from those of the typical VZV pneumonia, which can be potentially explained according to whether a patient has a primary infection or reactivation.

It is well known that VZV infection is a frequent complication of allogeneic bone marrow transplantation, and sometimes it has fatal consequences $(1,8)$. A previous Japanese report revealed that visceral VZV infection occurred in $0.8 \%$ of patients who underwent allogeneic hematopoietic stem cell transplantation and that the mortality rate was $20 \%$ (9). The pathogenesis of reactivation of VZV is still not fully understood but some studies have shown immunocompromised persons with impaired T-cell immunity (including recipients of organ or hematopoietic stem cell transplantations), those receiving immunosuppressive therapy, and those with lymphoma, leukemia, or HIV infection are at increased risk for herpes zoster (3). Prophylactic acyclovir is recommended after hematopoietic stem cell transplantation but the appropriate prophylactic dose and the optimal duration of acyclovir have not yet been clarified $(10,11)$. Our patient had taken $200 \mathrm{mg}$ of acyclovir prophylactically for 1.5 years after hematopoietic stem cell transplantation, and he had never experienced a VZV infection in the following 3 years. We had recommended that the patient take the VZV vaccine but he did not take it. We are also unaware of any history of varicella during his childhood. The administration of corticosteroids was started after he experienced an acute exacerbation of chronic interstitial pneumonia 1 year previously that may have increased the risk of reactivation of VZV (12). The glucocorticoid pulse therapy could have triggered the development of disseminated VZV disease in our patient although we are unable to confirm this possibility.

In terms of interstitial pneumonia, the association between acute exacerbation and viral infection remains unclear. An acute exacerbation of idiopathic pulmonary fibrosis has been defined as an acute, clinically significant respiratory failure, and, at the present time, finding triggers of acute exacerbation is important (13). We usually substitute this definition for other types of chronic interstitial pneumonia, and there have been some reports that viral infection may be a trigger of acute exacerbation of chronic interstitial pneumonia $(14,15)$. A previous Japanese study revealed that respiratory virus infections can be seen in $19.2 \%$ of the patients with an acute exacerbation of interstitial pneumonia, but that study did not report any VZV and a detailed association remains obscure (15). At the time of our patient's admission, we thought that our patient had an acute exacerbation of chronic interstitial pneumonia because he had a history of an acute exacerbation 1 year previously, and the radiographic findings were reasonable for considering this diagnosis. After starting treatment for the acute exacerbation, the characteristic skin lesion appeared on his chest and back. We then added acyclovir to his treatment regimen but his multiple organ failure rapidly worsened and he died. It is possible that VZV pneumonia could have triggered the acute exacerbation but his autopsy findings did not reveal any pathological changes that could explain such an acute exacerbation.

In summary, we experienced a case of fatal VZV pneumonia in a Japanese patient. The delay in the appearance of the characteristic skin lesions and atypical radiographic findings made it difficult to diagnose his VZV pneumonia. We think that those atypical findings may have been due to the difference between primary infection and reactivation but further analysis and accumulation of cases are needed to support this hypothesis. Physicians may have to consider the possibility of VZV pneumonia in immunocompromised patients, especially those with a medical history of hematopoietic stem cell transplantation.

The authors state that they have no Conflict of Interest (COI).

\section{References}

1. Feldman S. Varicella-zoster virus pneumonitis. Chest 106 (1 Suppl): 22S-27S, 1994.

2. Triebwasser JH, Harris RE, Bryant RE, Rhoades ER. Varicella 
pneumonia in adults. Report of seven cases and a review of literature. Medicine (Baltimore) 46: 409-423, 1967.

3. Cohen JI. Clinical practice: herpes zoster. N Engl J Med 369: 255263, 2013.

4. Stratman E. Visceral zoster as the presenting feature of disseminated herpes zoster. J Am Acad Dermatol 46: 771-774, 2002.

5. Yagi T, Karasuno T, Hasegawa T, et al. Acute abdomen without cutaneous signs of varicella zoster virus infection as a late complication of allogeneic bone marrow transplantation: importance of empiric therapy with acyclovir. Bone Marrow Transplant 25: 10031005,2000

6. Mirouse A, Vignon P, Piron P, et al. Severe varicella-zoster virus pneumonia: a multicenter cohort study. Crit Care 21: 137-147, 2017.

7. Rudinsky DM, Jordan K. Disseminated herpes zoster causing acute respiratory distress syndrome in an immunocompetent patient. BMJ Case Rep 2017: bcr-2017-220542, 2017.

8. Koc Y, Miller KB, Schenkein DP, et al. Varicella zoster virus infections following allogeneic bone marrow transplantation: frequency, risk factors, and clinical outcome. Biol Blood Marrow Transplant 6: 44-49, 2000.

9. Doki N, Miyawaki S, Tanaka M, et al. Visceral varicella zoster virus infection after allogeneic stem cell transplantation. Transpl Infect Dis 15: 314-318, 2013.

10. Kanda Y, Mineishi S, Saito T, et al. Long-term low-dose acyclovir against varicella-zoster virus reactivation after allogeneic hematopoietic stem cell transplantation. Bone Marrow Transplant 28: 689-692, 2011

11. Asano-Mori $\mathrm{Y}$, Kanda $\mathrm{Y}$, Oshima $\mathrm{K}$, et al. Long-term ultra-lowdose acyclovir against varicella-zoster virus reactivation after allogeneic hematopoietic stem cell transplantation. Am J Hematol 83: 472-476, 2008.

12. Sullivan NL, Eberhardt CS, Wieland A, et al. Characterization of virus-specific immune response during varicella zoster virus encephalitis in a young adult. Clin Infect Dis 69: 348-351, 2019.

13. Collard HR, Ryerson CJ, Corte TJ, et al. Acute exacerbation of idiopathic pulmonary fibrosis. An international working group report. Am J Respir Crit Care Med 194: 265-275, 2016.

14. Wootton SC, Kim DS, Kondoh Y, et al. Viral infection in acute exacerbation of idiopathic pulmonary fibrosis. Am J Respir Crit Care Med 183: 1698-1702, 2011.

15. Saraya T, Kimura H, Kurai D, et al. Clinical significance of respiratory virus detection in patients with acute exacerbation of interstitial lung disease. Respir Med 136: 88-92, 2018.

The Internal Medicine is an Open Access journal distributed under the Creative Commons Attribution-NonCommercial-NoDerivatives 4.0 International License. To view the details of this license, please visit (https://creativecommons.org/licenses/ by-nc-nd/4.0/).

(C) 2021 The Japanese Society of Internal Medicine Intern Med 60: 1077-1082, 2021 